\title{
Early Development of the Icon Molecule for Gene Therapy of Cancer and Macular Degeneration
}

\author{
Alan Garen* \\ Department Molecular Biophysics and Biochemistry, Yale University, USA \\ *Corresponding author: Alan Garen, Department Molecular Biophysics and Biochemistry, Yale University, USA. \\ To Cite This Article: Alan Garen. Early Development of the Icon Molecule for Gene Therapy of Cancer and Macular Degeneration. Am J Biomed Sci \\ \& Res. 2019 - 6(1). AJBSR.MS.ID.000992. DOI: 10.34297/AJBSR.2019.06.000992.
}

Received: 䟱 October 09, 2019; Published: 眥 November 04, 2019

\section{Mini-Review}

I conceived the Icon strategy for cancer immunotherapy in 1996 after reading a paper showing that Tissue Factor (TF) is expressed on the surface of vascular endothelial cells and tumor cells in invasive breast cancer but not in benign breast cancer [1]. Although it was not tested in this paper, I assumed TF also is not expressed on the surface of vascular endothelial cells in normal tissues and it might provide a specific marker for the tumor vasculature. TF is a cell-surface transmembrane receptor containing an extracellular domain that binds factor VII (fVII) with high affinity and specificity, initiating the blood coagulation pathway [2] (Figure 1). TF exists as an integral membrane protein in the intact wall of normal blood vessels, preventing contact with fVII circulating in the blood, in contrast to tumor blood vessels in which the vessel wall is damaged and leaky [3], exposing TF to fVII. Thus, TF could serve as a specific molecular target and fVII as a specific targeting molecule for the pathological neovasculature (PNV). Accordingly, I designed the Icon as a heavy-chain VH immunoconjugate antibody [4] composed of factor VII as the targeting domain attached either to a mouse or human Fc domain (Figure 2), similar to a Camelid antibody composed of heavy-chain dimers devoid of light chains [5]. A mutation was introduced into the active site of fVII to inhibit the initiation of the blood coagulation pathway, which might result in pathological blood clots [6].

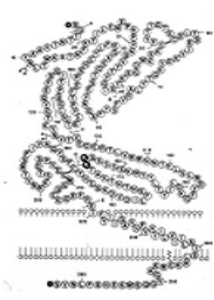

A

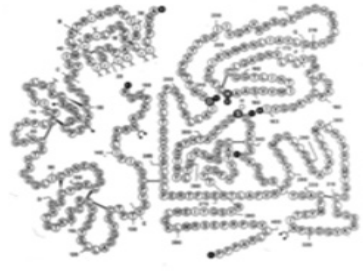

$\mathbf{B}$

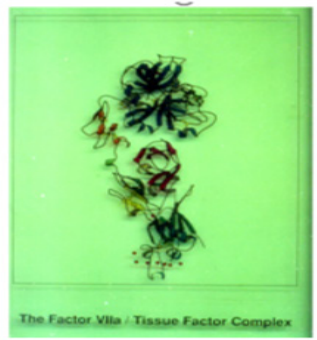

C

Figure 1: A. Diagram of (TF); B. Diagram of (fVIla); C. Structure of TF-fVla complex (2).

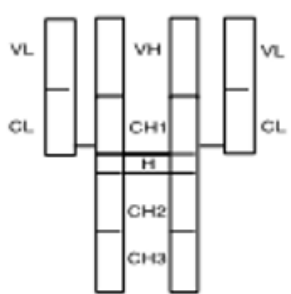

Human IgG1 antibody

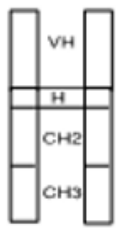

Camelid IgG1 antibody

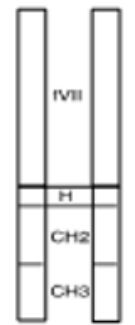

fVII/Fc Icon

Figure 2: Diagram of a Human IgG1 antibody, Camelid IgG1 antibody, and fVII/Fc Icon. 
In 1997 Zhiwei Hu joined my laboratory after receiving his doctorate from Hunan Medical University, and together with Ying Sun we demonstrated the efficacy and safety of the Icon in eradicating a human tumor xenograft in a mouse gene therapy model [7-9]. The Icon cDNA was incorporated into a replicationdefective adenovirus vector (AV) that was administered either by intratumor or intravenous injections. The AV-infected cells synthesized and secreted the Icon protein into the blood stream, resulting in binding of the Icon to TF on the tumor PNV and tumor cells, causing an immune attack by natural killer cells that eradicated the tumor PNV and tumor cells without evidence of damage to normal tissues. Of special significance was an experiment using a mouse model of metastasis containing two separate human tumor xenografts: one xenograft was injected with the Icon adenovirus vector, resulting in a cytolytic immune attack not only against the injected tumor xenograft but also against the distant noninjected tumor xenograft [9]. Yale University applied for a patent of the Icon on June 14, 2000 (US14216199P), which was granted on August 2, 2005 (US6924359B1), and the Icon technology was licensed to a start-up company called Iconics Therapeutics (IT). Our next Icon project concerned the exudative or wet form of macular degeneration (WMD), a major cause of vision impairment and blindness associated with aging [10]. wMD involves the PNV in the choroidal tissue located behind the retina, which invades and damages the macular region of the retina. As we had shown that the Icon binds to TF specifically in the PNV of solid tumors causing eradication the PNV, I considered that the Icon might have a similar effect on the choroidal PNV. In 2001 I contacted Dr. Henry Kaplan in the Department of Ophthalmology and Visual Sciences, University of Louisville, proposing a collaboration to test the Icon in his mouse model of laser-induced wMD. Our results showed that intraocular or intravenous injection of Icon protein or an adenoviral vector carrying the Icon cDNA caused eradication of the choroidal PNV without evidence of collateral damage to the normal choroidal and retinal vasculature $[11,12]$. Similar results were obtained in a pig model of wMD [13]. Experiments on photodynamic therapy with the Icon also were initiated in my laboratory and later continued by Zhiwei $\mathrm{Hu}$ [13].

Although most of our experiments of the Icon for treatment of solid tumors and wMD involved gene therapy using an adenovirus vector to deliver the Icon cDNA, gene therapy trials were put on hold following the tragic death of Jessie Gelsinger soon after receiving on September 13, 1999 an intravenous injection of an exceptionally high dose of a replication-deficient adenoviral vector carrying the normal OTC gene to treat his partial ornithine transcarbamylase (OTC) deficiency [14]. This procedure resulted in a massive immune response to the adenoviral vector causing formation of blood clots. Instead of using a gene therapy protocol, IT decided to produce the Icon protein ex vivo for injection in vivo. However, having failed to produce sufficient active Icon protein for treating cancer, IT has focused on treating WMD by intraocular injections of the Icon protein, which should be more efficacious for treating wMD than current protocols involving intraocular injections of antibodies against VEGF or VEGF receptors [15]. VEGF antibodies inhibit the development of the choroidal PNV and require continuing injections, in contrast to the Icon that eradicates the choroidal PNV [16]. Clinical trials of the Icon for wMD organized by IT are in progress [17]. To continue developing the Icon gene therapy approach for treating solid tumors, I proposed in 2000 a collaboration with Dr. Albert Deisseroth, who was then Chief of Medical Oncology at Yale University School of Medicine, to work with Zhiwei Hu and me on arranging a clinical trial of the Icon for treating melanoma by intratumor injections of a replication-incompetent adenovirus vector carrying the Icon cDNA. After Deisseroth left Yale in 2002 to become President of the Sidney Kimmel Cancer Center (SKCC) in La Jolla, California, he and I were Principal Investigators on a Program Project Grant to the SKCC to begin this project. We arranged with Molecular Medicine Biosciences (now known as SFAC Pharma in San Diego) to produce the Icon adenovirus vector under GMP conditions, but the final production could not be used clinically because of contamination with replication-competent recombinant adenoviruses, and the project could not be completed. The key advantages of the Icon gene therapy protocol for treating solid tumors, notably that the Icon appears to be efficacious for treating all types of solid tumors by eradicating the PNV and associated tumor without collateral damage to the normal vasculature, encourages further testing of other vectors $[18,19]$ that might avoid the initial problems associated with an adenovirus vector.

\section{References}

1. Contrino J, Hair G, Kreutzer DL, Rickles FR (1996) In situ detection of tissue factor in vascular endothelial cells: correlation with the malignant phenotype of human breast disease. Nat Med 2(2): 209-215.

2. Banner DW, D'Arcy A, Chène C, Winkler FK, Guha A, et al. (1996) The crystal structure of the complex of blood coagulation factor VIIa with soluble tissue factor. Nature 380(6569): 41-46.

3. Forster JC, Harriss Phillips WM, Douglass MJ, Bezak E, et al. (2017) A review of the development of tumor vasculature and its effects on the tumor microenvironment. Hypoxia (Auckl) 5: 21-32.

4. Cai X, A Garen (1996) A melanoma specific VH antibody cloned from a fusion phage library of a vaccinated melanoma patient. Proc Natl Acad Sci U S A 93(13): 6280-6285.

5. Hamers Casterman C, Atarhouch T, Muyldermans S, Robinson G, Hamers C, et al. (1993) Naturally occurring antibodies devoid of light chains. Nature 363(6428): 446-448.

6. Dickinson CD, CR Kelly, W Ruf (1996) Identification of surface residues mediating tissue factor binding and catalytic function of the serine protease factor VIIa. Proc Natl Acad Sci U S A 93(25): 14379-14384.

7. Hu Z, Y Sun, A Garen (1999) Targeting tumor vasculature endothelial cells and tumor cells for immunotherapy of human melanoma in a mouse xenograft model. Proc Natl Acad Sci USA 96(14): 8161-8166.

8. Hu Z, A Garen (2000) Intratumoral injection of adenoviral vectors encoding tumor-targeted immunoconjugates for cancer immunotherapy. Proc Natl Acad Sci USA 97(16): 9221-9225.

9. Hu Z, A Garen (2001) Targeting tissue factor on tumor vascular endothelial cells and tumor cells for immunotherapy in mouse models of prostatic cancer. Proc Natl Acad Sci USA 98(21): 12180-12185. 
10. Cheung LK, A Eaton (2013) Age-related macular degeneration. Pharmacotherapy 33(8): 838-855.

11. Bora PS, Hu Z, Tezel TH, Sohn JH, Kang SG, et al. (2003) Immunotherapy for choroidal neovascularization in a laser-induced mouse model simulating exudative (wet) macular degeneration. Proc Natl Acad Sci USA 100(5): 2679-2684.

12. Schubert C (2003) Meddling in macular degeneration. Nat Med 9(4): 396.

13. Hu Z, Oleinick N, Hamblin MR (2014) Photodynamic Therapy as an Emerging Treatment Modality for Cancer and Non-Cancer Diseases. J Anal Bioanal Tech S1: e001.

14. Tezel TH, Bodek E, Sönmez K, Kaliappan S, Kaplan HJ, et al. (2007) Targeting tissue factor for immunotherapy of choroidal neovascularization by intravitreal delivery of factor VII-Fc chimeric antibody. Ocul Immunol Inflamm 15(1): 3-10.
15. Lehrman S (1999) Virus treatment questioned after gene therapy death. Nature 401(6753): 517-518.

16. Volz C, D Pauly (2015) Antibody therapies and their challenges in the treatment of age-related macular degeneration. Eur J Pharm Biopharm 95(Pt B): 158-172.

17. John A Wells, Gonzales CR, Berger BB, Gonzalez VH, Sippy BD, et al. (2018) A Phase 1, Open-Label, Dose-Escalation Trial to Investigate Safety and Tolerability of Single Intravitreous Injections of ICON-1 Targeting Tissue Factor in Wet AMD. Ophthalmic Surg Lasers Imaging Retina 49(5): 336-345.

18. Lundstrom K (2018) Viral Vectors in Gene Therapy. Diseases 6(2): 42.

19. Yin H, Kanasty RL, Eltoukhy AA, Vegas AJ, Dorkin JR, et al. (2014) Nonviral vectors for gene-based therapy. Nat Rev Genet 15(8): 541-55. 